\title{
Ringed Structures of the HD 163296 Protoplanetary Disk Revealed by ALMA
}

\author{
Andrea Isella, ${ }^{1, *}$ Greta Guidi, ${ }^{2}$ Leonardo Testi, ${ }^{2,3}$ Shangfei Liu, ${ }^{1,4} \mathrm{Hui} \mathrm{Li},{ }^{4}$ Shengtai Li, ${ }^{4}$ Erik Weaver, ${ }^{1}$ \\ Yann Boehler, ${ }^{1}$ John M. Carperter, ${ }^{5}$ Itziar De Gregorio-Monsalvo, ${ }^{5}$ Carlo F. Manara, ${ }^{6}$ Antonella Natta, ${ }^{7,2}$ \\ Laura M. Pérez, ${ }^{8}$ Luca Ricci, ${ }^{9}$ Anneila Sargent,${ }^{10}$ Marco Tazzari, ${ }^{3}$ and Neal Turner ${ }^{11}$ \\ ${ }^{1}$ Department of Physics and Astronomy, Rice University, 6100 Main Street, MS-108, Houston, Texas 77005, USA \\ ${ }^{2}$ INAF/Osservatorio Astrofisico di Arcetri, Largo E. Fermi 5, I-50125 Firenze, Italy \\ ${ }^{3}$ ESO, Karl Schwarzschild str. 2, 85748 Garching bei Mnchen, Germany \\ ${ }^{4}$ Theoretical Division, Los Alamos National Laboratory, Los Alamos, New Mexico 87545, USA \\ ${ }_{5}^{5}$ Joint ALMA Observatory (JAO), Alonso de Cordova 3107 Vitacura, Santiago de Chile, Chile \\ ${ }^{6}$ Scientific Support Office, Directorate of Science, European Space Research and Technology Centre (ESA/ESTEC), \\ Keplerlaan 1, 2201 AZ Noordwijk, Netherlands \\ ${ }^{7}$ School of Cosmic Physics, Dublin Institute for Advanced Studies, 31 Fitzwilliams Place, 2 Dublin, Ireland \\ ${ }^{8}$ Max-Planck-Institut fr Radioastronomie Bonn, auf dem Hgel 69, D-53121 Bonn, Germany \\ ${ }^{9}$ Harvard-Smithsonian Center for Astrophysics, 60 Garden Street, Cambridge, Massachusetts 02138, USA \\ ${ }^{10}$ Division of Physics, Mathematics and Astronomy, California Institute of Technology, Pasadena, California 91125, USA \\ ${ }^{11}$ Jet Propulsion Laboratory, California Institute of Technology, Pasadena, California 91109, USA
}

(Received 5 July 2016; revised manuscript received 11 October 2016; published 12 December 2016)

We present Atacama Large Millimeter and Submillimeter Array observations of the protoplanetary disk around the Herbig Ae star HD 163296 that trace the spatial distribution of millimeter-sized particles and cold molecular gas on spatial scales as small as 25 astronomical units (A.U.). The image of the disk recorded in the $1.3 \mathrm{~mm}$ continuum emission reveals three dark concentric rings that indicate the presence of dust depleted gaps at about 60, 100, and 160 A.U. from the central star. The maps of the ${ }^{12} \mathrm{CO},{ }^{13} \mathrm{CO}$, and $\mathrm{C}^{18} \mathrm{O} J=2-1$ emission do not show such structures but reveal a change in the slope of the radial intensity profile across the positions of the dark rings in the continuum image. By comparing the observations with theoretical models for the disk emission, we find that the density of $\mathrm{CO}$ molecules is reduced inside the middle and outer dust gaps. However, in the inner ring there is no evidence of CO depletion. From the measurements of the dust and gas densities, we deduce that the gas-to-dust ratio varies across the disk and, in particular, it increases by at least a factor 5 within the inner dust gap compared to adjacent regions of the disk. The depletion of both dust and gas suggests that the middle and outer rings could be due to the gravitational torque exerted by two Saturn-mass planets orbiting at 100 and 160 A.U. from the star. On the other hand, the inner dust gap could result from dust accumulation at the edge of a magnetorotational instability dead zone, or from dust opacity variations at the edge of the $\mathrm{CO}$ frost line. Observations of the dust emission at higher angular resolution and of molecules that probe dense gas are required to establish more precisely the origins of the dark rings observed in the HD 163296 disk.

DOI: $10.1103 /$ PhysRevLett.117.251101

Introduction.-After centuries of speculation about planets orbiting stars other than our Sun, the discovery of an extrasolar planet with Jupiter-like mass came in 1995 [1]. To date, the most fruitful methods of planetary searches have been transit and radial velocity surveys [2,3], especially Kepler's transit surveys [4]. However, the discoveries of more than 2000 exoplanet candidates [5], mostly gas giants and super-Earths, have raised even more challenges to understanding such systems. For example, while giant planets are believed to form 0.1-10 A.U. from the central star [6], the newly discovered orbital radii cover a much broader range, 0.01-100 A.U. This suggests either a surprisingly large mobility of planets or a fundamental flaw in current planetary formation models.

Recently, the unprecedented angular resolution and sensitivity of Atacama Large Millimeter and
Submillimeter Array (ALMA) observations have led to the discovery of ring-shaped structures in the $\mathrm{mm}$ continuum emission from the HL Tau and TW Hya protoplanetary disks $[7,8]$. While the origin of these structures is still debated, it is likely that they are related to the formation of planets, and hence may provide long-awaited observational constraints on the processes responsible for planetary system assembly and evolution.

A number of explanations for the presence of rings in the continuum emission in protoplanetary disks have been put forward. These include the aggregation of solids at the outer edge of disk regions characterized by low turbulence (e.g., [9-14]), variations in the dust opacity at the frost line of volatile elements $[15,16]$, and gravitational perturbations caused by yet unseen giant planets [17]. In the first two cases, the observed structures might aid the formation of 
Continuum flux density [Jy/beam]
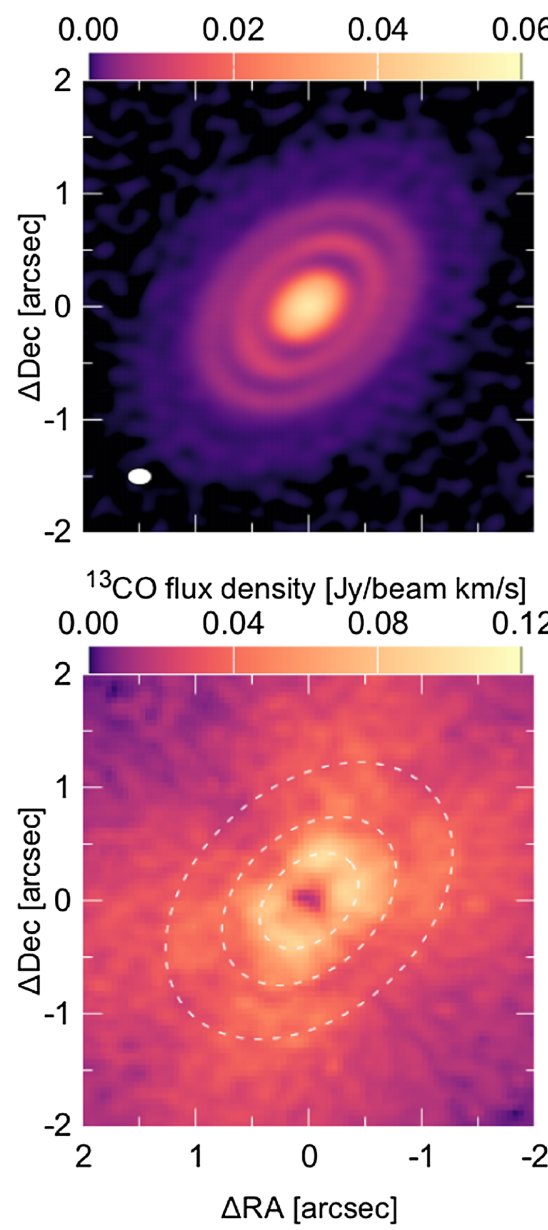

${ }^{12} \mathrm{CO}$ flux density [Jy/beam $\mathrm{km} / \mathrm{s}$ ]

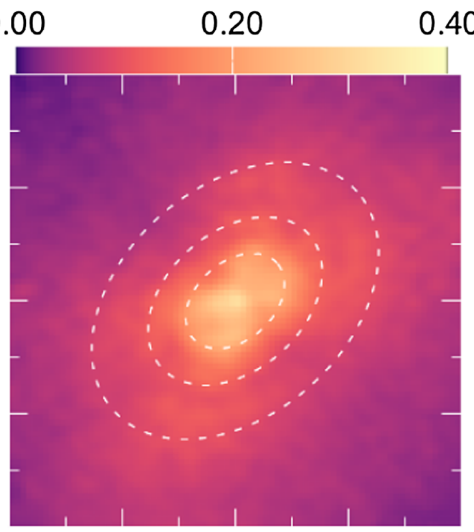

$\mathrm{C}^{18} \mathrm{O}$ flux density [Jy/beam $\mathrm{km} / \mathrm{s}$ ]
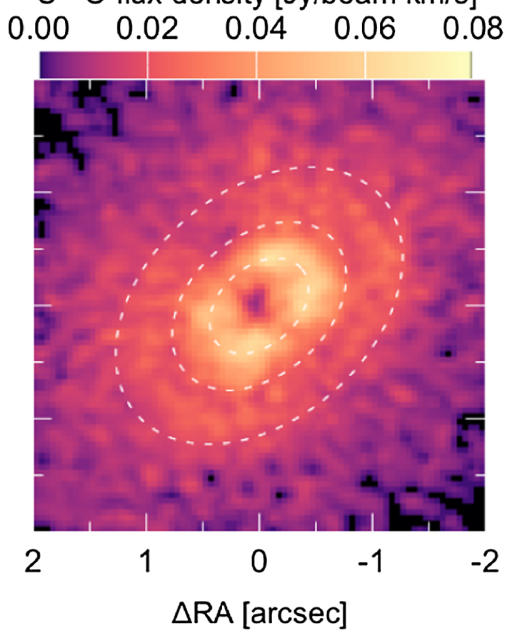
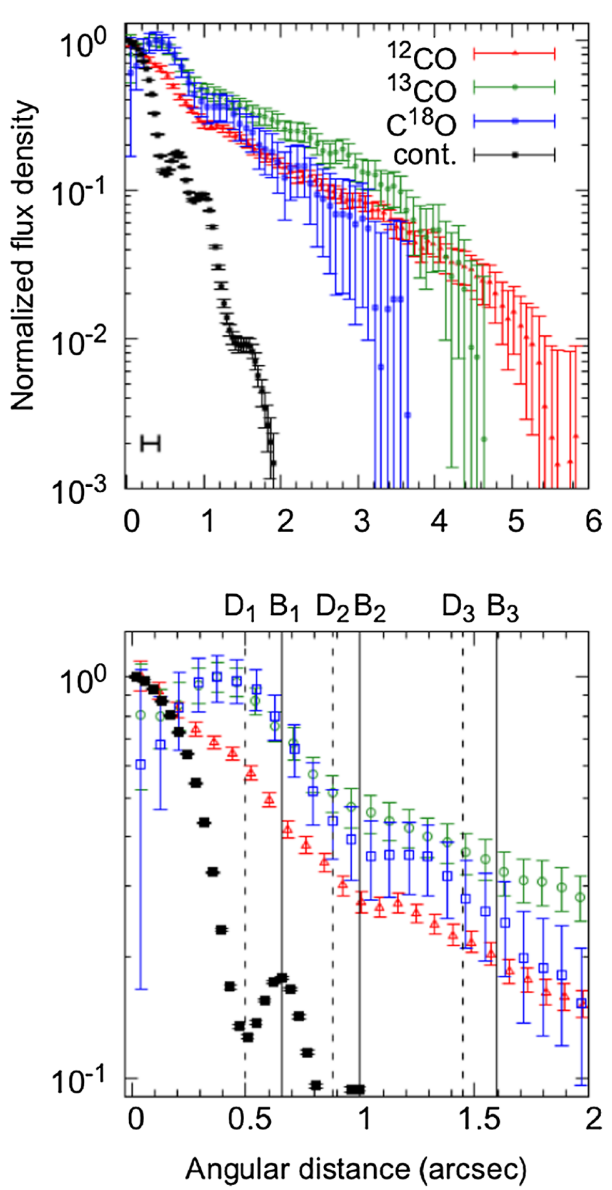

FIG. 1. ALMA images of the HD 163296 disk emission recorded in $1.3 \mathrm{~mm}$ dust continuum (top left), ${ }^{12} \mathrm{CO}$ (top middle), ${ }^{13} \mathrm{CO}$ (bottom left), and $\mathrm{C}^{18} \mathrm{O}$ (bottom middle) $J=2-1$ line emission. The angular resolution of the observations, $0.22^{\prime \prime} \times 0.14^{\prime \prime}$, is indicated by the filled white ellipse in the continuum image. The dashed ellipses in the CO maps indicate the locations of the dark rings seeing in the continuum map. Azimuthally averaged profiles, normalized to the peak intensities, are shown in the right panels. They are calculated by averaging on elliptical annuli with a position angle of $132^{\circ}$, an eccentricity of 0.7 , and a width equal to $1 / 4$ of the angular resolution of the observations. The error bars are calculated by dividing the root mean square noise of the observations (see Table I in the Supplemental Material [26]) by the square root of the number of independent beams in each annulus. The vertical lines indicate the position of the dark $(D)$ and bright $(B)$ rings observed in the continuum map. The horizontal bar in the top right panel indicates the angular resolution of the observations.

planetesimals by concentrating solid particles and preventing them from accreting onto the star $[18,19]$. In the last case, a circular gap is expected when a forming planet is orbiting in a disk. The orbital radius and mass of the planet can be inferred from the location and shape of this gap [17]. For HL Tau, it has been suggested that the observed ringed structure is due to the presence of Saturn-mass planets orbiting at several tens of A.U. from the central star [20]. This implies that giant planets can form on much shorter time scales, and at much larger distances from the central star, than predicted by current planet formation models [6].

The initial results of ALMA observations are promising but key questions remain unanswered. In particular, it is not clear if ringlike structures are common to all protoplanetary disks. ALMA high angular resolution observations targeted the most luminous disks first, and these may not be representative of the bulk of the disk population. Furthermore, dust continuum images do not probe the distribution of the circumstellar gas and thus cannot distinguish unambiguously between different formation processes. Addressing such questions requires a multipronged approach involving both large continuum surveys of nearby protoplanetary disks with ALMA and in-depth multiwavelength studies of the most relevant structures revealed.

In this Letter, we report on the structure of the HD 163296 disk as revealed by ALMA observations of the dust continuum emission and also line emission from three isotopologues of carbon monoxide, ${ }^{12} \mathrm{CO},{ }^{13} \mathrm{CO}$, and $\mathrm{C}^{18} \mathrm{O}$. HD 163296 is a young $(5 \mathrm{Myr})$ intermediate mass $\left(2.3 M_{\odot}\right)$ 
Herbig Ae star located at $122_{-13}^{+17} \mathrm{pc}$ from Earth [21]. The star is surrounded by a gaseous Keplerian disk with a radius of about 550 A.U. By contrast, mm-wave continuum emission arising from solid particles is confined within 250 A.U. from the star $[22,23]$. Previous ALMA observations showed that the continuum emission has a local maximum at about 90 A.U. [24], suggesting a dusty ring at a distance that corresponds to the frost line for $\mathrm{CO}$ molecules [25]. Recently, we have imaged the HD 163296 disk with ALMA at $0.2^{\prime \prime}$ angular resolution, a factor of 3 higher than previous observations and corresponding to a physical scale of 25 A.U. at the distance of the star. The observations reveal rings in the dust continuum emission similar to those observed toward HL Tau. They also reveal the morphology of the ${ }^{12} \mathrm{CO},{ }^{13} \mathrm{CO}$, and $\mathrm{C}^{18} \mathrm{O}$ emission on the same angular scales as the continuum. Here we show that the combination of continuum and molecular line observations offers stringent tests for several physical processes related to planet formation and planet-disk interaction.

Observations.-Figure 1 shows the images of the HD 163296 continuum emission together with those of the continuum-subtracted, spectrally integrated ${ }^{12} \mathrm{CO},{ }^{13} \mathrm{CO}$, and $\mathrm{C}^{18} \mathrm{O} J=2-1$ lines. The acquisition and calibration of the data are described in the Supplemental Material [26]. Also displayed are the radial intensity profiles across the disk calculated by averaging the flux density over elliptical annuli characterized by a position angle of $132^{\circ}$ and an eccentricity of 0.7 to correct for the disk inclination and orientation $[22,25]$.

The continuum emission is in first approximation centrosymmetric and confined to an angular radius of about $2^{\prime \prime}$ from the central star. It is also characterized by three pairs of low and high luminosity elliptical rings, labeled as $(D 1$, $B 1),(D 2, B 2)$, and $(D 3, B 3)$, where $(D 1, B 1)$ and $(D 3, B 3)$ are the innermost and outermost pairs, respectively. The aspect ratios of the rings correspond to an intrinsically circular disk inclined by $42^{\circ}$ with respect to the line of sight; $90^{\circ}$ corresponds to an edge-on orientation. The inclination agrees with previous measurements based on CO observations $[22,25]$. The radial profile of the dust continuum emission decreases by a factor of 1000 within $2^{\prime \prime}$ of the star. The ratios of the continuum intensity for each high or low luminosity ring pair are $1.6(B 1 / D 1), 1.3$ $(B 2 / D 2)$, and $1.08(B 3 / D 3)$. In the first approximation, the azimuthally averaged profile of the continuum flux density can be modeled by a combination of Gaussian functions, $f(\theta)=g(\theta)-g_{1}(\theta)-g_{2}(\theta)-g_{3}(\theta)$, where $g(\theta)=a e^{-(\theta / b)^{2}}$ and $g_{n}(r)=a_{n} e^{-\left[\left(\theta-\theta_{n}\right) / b_{n}\right]^{2}}$. This profile mimics that of a disk with gaps in the continuum flux density characterized by radius $\theta_{n}$ and width $b_{n}$. A nonlinear least-squares Marquardt-Levenberg fit of the observations returns $\theta_{1}=$ $(0.44 \pm 0.01)^{\prime \prime}, \theta_{2}=(0.81 \pm 0.01)^{\prime \prime}, \theta_{3}=(1.13 \pm 0.02)^{\prime \prime}$, and $\quad b=(0.76 \pm 0.02)^{\prime \prime}, \quad b_{1}=(0.23 \pm 0.01)^{\prime \prime}, \quad b_{2}=$ $(0.15 \pm 0.01)^{\prime \prime}, \quad b_{3}=(0.28 \pm 0.02)^{\prime \prime}$, with a reduced $\chi^{2} \sim 2$. A table with the best fit value of the Gaussian modeling is provided in the Supplemental Material [26].

Molecular emission is detected in all three CO isotopologues, and differs from the continuum in three main aspects. First, the $\mathrm{CO}$ intensity maps do not show any prominent ringed structure and, differently from the continuum, their radial profiles cannot be approximated by a simple mathematical function. Second, the ${ }^{13} \mathrm{CO}$ and $\mathrm{C}^{18} \mathrm{O}$ line intensities have local minima at the center of the disk, while both the continuum and the ${ }^{12} \mathrm{CO}$ line are centrally peaked. We ascribe the ${ }^{13} \mathrm{CO}$ or $\mathrm{C}^{18} \mathrm{O}$ central minima to an oversubtraction of the continuum emission, and not to a physical decrement of the gas density or temperature. This point is discussed in more detail in the Supplemental Material [26]. Third, the molecular emission is much more extended and decreases with the orbital radius more slowly than the continuum. In particular, the ${ }^{12} \mathrm{CO}$ emission decreases only to about $15 \%$ of its peak value at radius $2^{\prime \prime}$. Zooming into the central disk regions, as in the bottom right panel on Fig. 1, indicates that only mild changes in the $\mathrm{CO}$ emission occurs at the location of the continuum rings. A bump in ${ }^{12} \mathrm{CO}$ emission is observed near $D 1$ and a flattening of the slope of the emission can be seen near $B 2$ for all three isotologues, with the $\mathrm{C}^{18} \mathrm{O}$ emission increasing slightly between $1^{\prime \prime}$ and $1.2^{\prime \prime}$.

Data analysis.-In this section, we investigate the ringed structure observed in the continuum and the aforementioned morphological differences between continuum and molecular line emission. We do this by modeling the continuum and spectrally integrated line emission maps presented in Fig. 1. The analysis of the spectrally resolved line emission will be presented in a future work.

The continuum and line emission of a protoplanetary disk depend on the three-dimensional density and temperature distribution of dust and gas, the gas velocity, the dust opacity, the disk inclination, and the distance of the source. In recent years, the HD 163296 disk has been the subject of several studies whose results provide a starting point for our investigation. Following the methodology described in [23] and [27], we describe the surface density of gas and dust as

$$
\Sigma(r)=\Sigma_{c}\left(r / r_{c}\right)^{-\gamma} \exp \left[-\left(r / r_{c}\right)^{2-\gamma}\right],
$$

and the disk temperature as

$$
T(r, z)= \begin{cases}T_{a}(r, z)+\left[T_{m}(r)-T_{a}(r, z)\right]\left(\cos \frac{\pi z}{2 z_{q}(r)}\right)^{2 d(r)}, & \text { if }|z|<z_{q}(r) \\ T_{a}(r, z), & \text { otherwise. }\end{cases}
$$



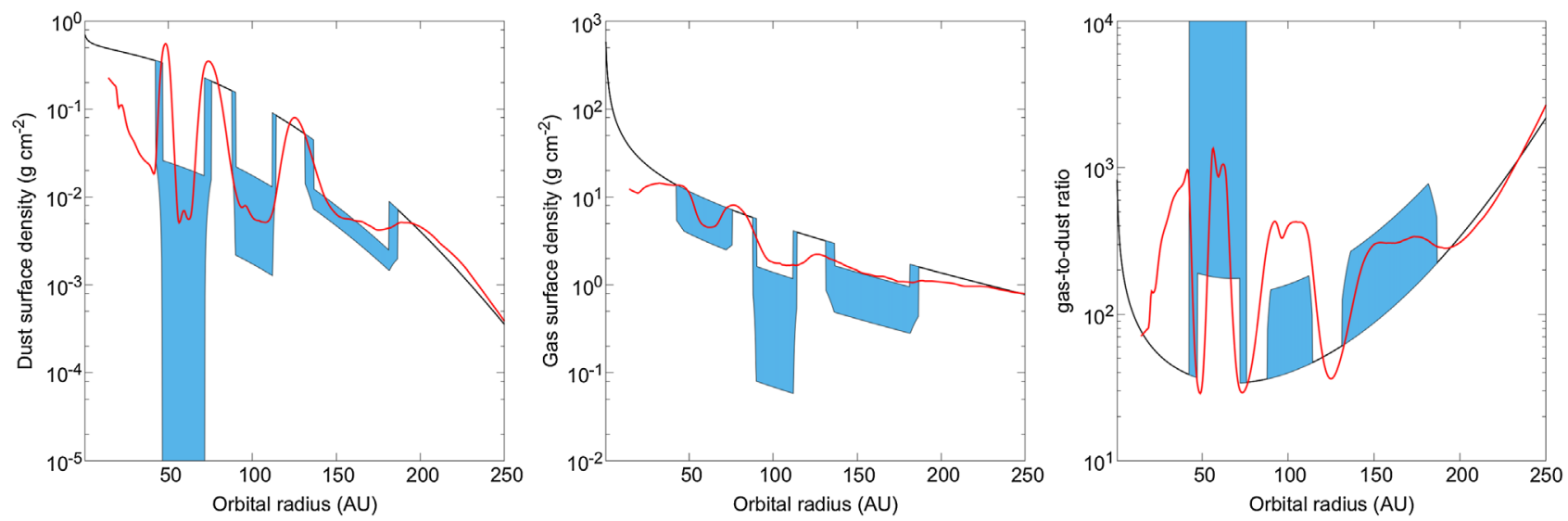

FIG. 2. Constraints on the dust and gas surface density (left and middle panels, respectively), and on the gas-to-dust ratio (right panel), derived by comparing ALMA observations of the HD163296's disk with synthetic models for the disk emission. The red solid curve indicates the prediction for a disk model perturbed by three planets with masses of $0.1,0.3$, and $0.3 M_{J}$ orbiting at 62,105 , and 160 A.U. from the central star.

In the latter equation, $T_{m}(r)=T_{m, 0}\left(r / r_{0}\right)^{-q_{m}}$ and $T_{a}(r, z)=T_{a, 0}\left(\sqrt{r^{2}+z^{2}} / r_{0}\right)^{-q_{a}}$ are the temperature of the disk midplane and surface layer, respectively. The temperatures of these two layers are smoothly connected through the functions $d(r)=d_{0}\left(r-r_{0}\right)+c$ and $z_{q}(r)=z_{q, 1}\left(r / r_{1}\right)^{q_{z}} e^{-\left(r / r_{2}\right)^{2}}$. This rather complex parametrization of the disk temperature mimics the thermal structure of an externally illuminated disk and enables us to generate synthetic models for the dust and gas emission while avoiding calculating the disk temperature using time-consuming radiative transfer models.

Using Eqs. (1) and (2), and the procedure described in the Supplemental Material [26], we find that the dust emission measured at angular radii less than $0.3^{\prime \prime}$ and lager than $1.8^{\prime \prime}$ is consistent with a dust surface density characterized by $\gamma=0.1, r_{c}=90$ A.U., $\Sigma_{c}=0.42 \mathrm{~g} \mathrm{~cm}^{-2}$. The low value of $\gamma$ implies that the dust density within about 90 A.U. from the star scales with the radius approximately as a Gaussian. This result is consistent with the parametric fitting of the dust continuum emission described in the previous section.

Applying the same procedure to the ${ }^{12} \mathrm{CO},{ }^{13} \mathrm{CO}$, and $\mathrm{C}^{18} \mathrm{O}$ emission, we find gas surface densities characterized by $\gamma=0.8, r_{c}=165$ A.U., $\Sigma_{c}\left({ }^{12} \mathrm{CO}\right)=1.6 \times 10^{-3} \mathrm{~g} \mathrm{~cm}^{-2}$, $\Sigma_{c}\left({ }^{13} \mathrm{CO}\right)=2.3 \times 10^{-5} \mathrm{~g} \mathrm{~cm}^{-2}$, and $\Sigma_{c}\left(\mathrm{C}^{18} \mathrm{O}\right)=2.9 \times$ $10^{-6} \mathrm{~g} \mathrm{~cm}^{-2}$. Our modeling suggests that (i) the ${ }^{12} \mathrm{CO} /{ }^{13} \mathrm{CO}$ and ${ }^{12} \mathrm{CO} / \mathrm{C}^{13} \mathrm{O}$ density ratios are radially constant, (ii) the $\mathrm{CO} /$ dust ratio changes with the orbital radius, and (iii) that the disk thermal structure is characterized by $T_{m}(r)=24 \mathrm{~K}(r / 100 \text { A.U. })^{-0.5}$ and $T_{a}(r, z)=$ $68 \mathrm{~K}\left(\sqrt{r^{2}+z^{2}} / 100 \text { A.U. }\right)^{-0.6}$.

However, disk models characterized by a smooth density profile predict too much emission in the region where rings are seen in dust continuum. To correct for this excess, we introduce circular gaps in the dust and gas surface density. Each gap is described by three parameters: the distance between the center of the gap and the star $(R)$, the width of the gap $(W)$, and the ratio between the surface density inside and outside the gap $(\Delta)$. We calculate synthetic images for the dust continuum, ${ }^{12} \mathrm{CO},{ }^{13} \mathrm{CO}$, and $\mathrm{C}^{18} \mathrm{O}$ $J=2-1$ emission for different values of the gap parameters $(R, W, \Delta)$, and compare them to the observations. Figure 2 shows the set of models that better reproduce the observations, while the modeling procedure is described in the Supplemental Material [26].

The colored area in the left panel of Fig. 2 shows the values of the dust surface density compatible with the observations. The dark rings observed in the continuum map are compatible with the presence of three partially dust depleted gaps centered at $R_{1}=60$ A.U., $R_{2}=100$ A.U., and $R_{3}=160$ A.U.. Given the finite angular resolution of the observations, there is a degeneracy between the gap width $\mathrm{W}$ and dust depletion $\Delta^{d}$. We find that the narrowest gaps compatible with the observations have widths of $W_{1}=25$ A.U., $W_{2}=22$ A.U., and $W_{3}=45$ A.U.. The dust depletion factors inside the gaps are $\Delta_{1}^{d}>100$, $\Delta_{2}^{d}=70$, and $\Delta_{3}^{d}=6$. The widest gaps compatible with the observations have widths of $W_{1}=33$ A.U., $W_{2}=26$ A.U., and $W_{3}=55$ A.U., and depletion factors $\Delta_{1}^{d}=13, \Delta_{2}^{d}=7$, and $\Delta_{3}^{d}=3.6$. The dust surface density inside the inner gap is $\lesssim 3 \times 10^{-2} \mathrm{~g} \mathrm{~cm}^{-2}$, while it varies between $3 \times 10^{-3}-3 \times 10^{-2} \mathrm{~g} \mathrm{~cm}^{-2}$ and $4 \times 10^{-3}-7 \times$ $10^{-3} \mathrm{~g} \mathrm{~cm}^{-2}$ in the middle and outer gaps, respectively. The ratios between the half-width and the radius of the gaps are between 0.21 and 0.28 for the innermost gap, $0.1-13$ for the middle gap, and 0.14-0.17 for the outermost gap.

The central panel of Fig. 2 shows the constraints on the gas $\left(\mathrm{H}_{2}\right)$ surface density derived from the $\mathrm{CO}$ observations adopting a cosmic molecular abundance $n\left({ }^{12} \mathrm{CO} / \mathrm{H}_{2}\right)=$ $5 \times 10^{-5}$ [28]. In our analysis, we assume that the gaps in the dust and $\mathrm{CO}$ have the same width and radius, and explore different values for the depletion factor $\Delta^{\mathrm{CO}}$. Inside 
the inner dust gap, we measure $\Delta_{1}^{\mathrm{CO}}$ between 0 and 2.5 . This implies that CO molecules are at least five times less depleted than the dust. In the middle and outer gaps, we measure $\Delta_{2}^{\mathrm{CO}}$ between 3.5 and 70 and $\Delta_{3}^{\mathrm{CO}}$ between 1.8 and 6 , respectively. These latter values are similar to those inferred for the dust.

Finally, the right panel of Fig. 2 shows the gas-to-dust ratio obtained by combining the results for the dust and gas surface density. The gas-to-dust ratio increases from about 40 at 100 A.U. to more than 1000 for $r>250$ A.U.. The gas-to-dust ratio inside the first dust gap is at least five times larger than outside the gap. Such an increase results from the discrepancy between the depletion in dust and CO. Inside the middle and outer dust gaps the gas-to-dust ratio varies by less than a factor of 2 . Our model also suggests that the gas-to-dust ratio might increase for $r<50$ A.U.. However, this result should be taken with caution because both the dust and $\mathrm{CO}$ emission inside this radius are optically thick and the corresponding surface densities are affected by a large uncertainty.

Discussion.-To date, the HD 163296 disk is the third system known to host multiple rings in the dust continuum emission, but it is the only one for which we have succeeded in mapping the molecular gas emission at the same angular resolution of the continuum. As mentioned above, such ringed structures are thought to be related to the formation of planets, but it is unclear whether they trace an early stage of planet assembly or whether they result from the interaction with already formed planets. In this section we discuss how measuring the spatial distribution of both gas and dust is key to address this question.

Disk-planet interaction: The interaction between disks and planets is based on the notion that the gravitational field of a planet exerts a tidal torque on the circumstellar material, which allows the transfer of angular momentum between the planet and the disk (see, e.g., [29]). The consequences are twofold: on one hand, the planet is predicted to migrate; on the other hand, the density waves launched by the planet are expected to alter the disk surface density. If the planet gravity exceeds the stellar gravity across the vertical extent of the disk, then the planet opens a circular gap in the disk surface density [17]. The radius, width, and level of gas and dust depletion within the gap carry information about the planet and disk properties.

Numerical simulations show that the width $W$ of a gap opened by a planet should depend on the planet mass $M_{p}$ and orbital radius $R_{p}$ following the relation

$$
W \sim C \times R_{p}\left(\frac{M_{p}}{3 M_{\star}}\right)^{1 / 3},
$$

where the proportionality constant $C$ varies between 4 and $8[17,30,31]$. In the case of HD 163296, the gap widths derived in the previous section imply planet masses between 0.5 and $2 M_{J}$ for the innermost planet, 0.05 and
$0.3 M_{J}$ for the middle planet, and 0.15 and $0.5 M_{J}$ for the outer planet. In addition, the disk viscosity can affect both the gap width and depth [32]. Various models have predicted that, at equilibrium, the depth of a gap opened by a planet in the gas surface density should depend on the planet mass through the relation [33]

$$
\frac{M_{p}}{M_{\star}}=5 \times 10^{-4}(\Delta-1)^{1 / 2}\left(\frac{h}{0.1}\right)^{5 / 2}\left(\frac{\alpha}{10^{-3}}\right)^{1 / 2},
$$

where $h$ is the gas disk aspect ratio, $\Delta$ is the gas depletion ratio, and $\alpha$ is the Shakura-Sunyaev viscosity parameter [34]. This relation indicates that generally a lower disk viscosity and aspect ratio results in a deeper gap for a fixed planet mass.

In the case of HD 163296, we use our disk temperature model derived from the observations to calculate a disk aspect ratio of 0.05 at 60 A.U., 0.065 at 100 A.U., and 0.07 at 160 A.U. By combining Eqs. (3) and (4) we estimate the viscosity parameter $\alpha$. We obtain $\alpha \gtrsim 2 \times 10^{-2}$ in the inner gap, $10^{-6}<\alpha<5 \times 10^{-3}$ in the middle gap, and $10^{-4}<$ $\alpha<7 \times 10^{-3}$ in the outer gap. The larger $\alpha$ of the inner gap results from the fact that low or no gas depletion is observed despite a dust gap width that is larger than those of the other two dust gaps. These heuristic considerations work reasonably well for the middle and outer gaps but the inner gap presents additional challenges. The gas-to-dust ratio is the largest for this gap, which for a given planet mass requires a relatively low viscosity (e.g., [19]), in contrast to the value derived above. This discrepancy suggests that additional processes might be happening around the first gap, including the possibility of having multiple planets.

To better understand the effect of planet perturbations on the structure of the HD 163296 disk, we have compared the gas and dust surface density derived from ALMA observations with hydrodynamic simulations of the planet-disk interaction. The setup of the simulations is described in the Supplemental Material [26]. The red line in Fig. 2 shows the dust and gas surface density for a disk perturbed by three planets with masses of $0.1 M_{J}, 0.3 M_{J}$, and $0.3 M_{J}$ orbiting at 62, 105, and 160 A.U. from the star. The surface density profiles were extracted after 2000 orbits of the innermost planet corresponding to $7 \times 10^{5} \mathrm{yr}$.

We find that the gas and dust surface density measured across the middle and outer gaps are compatible with perturbations from Saturn-mass planets and a disk viscosity parameter $10^{-3}<\alpha<10^{-2}$. The planets carve rather shallow and axisymmetric gaps in the gas surface density, whereas the gaps in the dust are deeper due to migration of solid particles toward the edges of the gas gaps. As a consequence, the gas-to-dust ratio inside the gaps formed by the planets increases with respect to the surrounding disk regions.

Our simulations show that perturbations from a single planet cannot explain the properties of the inner gap. The 
width of the gap generated by a $0.1 M_{J}$ planet is narrower than that suggested by the observations. A more massive planet would make the gap wider, but the gap would be too depleted in gas. Increasing both the planet mass and the disk viscosity would make the gap wider and shallower, but a larger viscosity would cause the gas-to-dust ratio inside the gap to drop well below 5. As a possible solution, we argue that the inner gap might be created by multiple planets between 50 and 70 A.U. For example, two Saturnmass planets orbiting around 55 and 65 A.U. might create a joint gap consistent with the observations, though the stability of such a configuration remains to be investigated (see, e.g., [35]). However, observations with higher angular resolution are required to test this hypothesis.

MRI dead zone: An ionized Keplerian disk embedded in a weak magnetic field is predicted to be subject to the magnetorotational instability (MRI) [36]. MRI should generate turbulent motions capable of transporting angular momentum outward, therefore allowing the circumstellar material to accrete onto the star. If the gas ionization varies with the orbital radius, then the mass flow rate throughout the disk is not constant and gas and dust can pile up forming ringed structures.

There are two main transitions in the gas ionization in a disk characterized by a monotonically decreasing surface density. The first happens at a fraction of A.U. from the star where the gas temperature exceed $1000 \mathrm{~K}$ and the thermal ionization is strong. The ionized region is predicted to have high turbulence velocities and quickly accrete onto the central star, possibly leading to the formation of a large central hole [37]. At larger distances the ionization is nonthermal and caused mostly by stellar ultraviolet and $\mathrm{x}$-ray photons and interstellar cosmic rays, which are absorbed over columns of $0.1,10$, and $100 \mathrm{~g} \mathrm{~cm}^{-2}$, respectively [38-40]. The ionized regions experience MRI, which leads to turbulent speeds that increase with height above the disk midplane in proportion to the Alfven speed, reaching the local sound speed above several disk scale heights [41,42]. At surface densities above about $10 \mathrm{~g} \mathrm{~cm}^{-2}$, the disk midplane is expected to be mostly neutral and quiescent. In the outermost disk regions, for $\Sigma<10 \mathrm{~g} \mathrm{~cm}^{2}$, the disk midplane returns to be partially ionized. The sharp gradient in turbulence, and henceforth in mass flow rate, at the interface between the MRI dead zone and the MRI active midplane is predicted to lead to a pileup of dust and gas, which might appear as an emission ring once observed with ALMA [43].

Magnetohydrodynamics simulations of protoplanetary disks predict that the gas surface density at the outer edge of a MRI dead zone might increase by a factor of a few compared to the surrounding regions [43]. The increase in gas density creates gas pressure maxima that might trap dust particles, therefore augmenting the radial variation in the dust surface density. Our measurement of the gas surface density of the HD 163296 disk (central panel of
Fig. 2) indicates that the MRI dead zone might extend to about 60-100 A.U. from the central star. This distance is similar to the radius of the first bright continuum ring, suggesting that this process might perhaps be involved in the ring formation. Furthermore, the large gas-to-dust ratio measured inside the inner dust gap implies low viscosity. This is consistent with the presence of a MRI dead zone. The middle and outer dust gaps at 100 and 160 A.U. from the star do not seem to be compatible with this scenario, though.

Phase transition of volatile elements: The phase transition from solid to gas of molecules such as $\mathrm{H}_{2} \mathrm{O}$ and $\mathrm{CO}$ is predicted to alter the coagulation and fragmentation properties of dust grains, possibly leading to the accumulation of dust particles in ringlike structures. Dust grains located in the cold regions of protoplanetary disks are expected to be covered by ice mantles, which make them more sticky compared to bare grains and more prone to growth in size as a result of collisions. As the size of a dust grain increases, so does the drag caused by the aerodynamic friction with the circumstellar gas. As a result of this process, larger icy grains are predicted to drift inward toward warmer disk regions. Eventually they cross the frost lines relative to the molecules that form their ice mantles, which then evaporate. When this happens, the grain size shrinks affecting both its opacity and the gas drag. The combined result is an increase in the dust opacity right behind the frost line, which might result in the formation of bright emission rings in the microwave continuum emission [15,44].

A second effect of the evaporation of volatile elements from dust grains is described by the so-called "sintering" model [16], which postulates that the grains are fractal aggregates of smaller particles. Outside each volatile's frost line, the aggregates are covered by a layer of ice. As inward drifting particles approach the frost line, the ice molecules become mobile and migrate to the necks between the component particles, making rolling and sliding difficult. This reduces the internal degrees of freedom available to take up excess energy when aggregates collide. The aggregates become hard but brittle, and break apart easily in collisions. The smaller fragments drift more slowly, enhancing the dust surface density near the snow line and causing radial variations in the dust opacity [16].

In general, the snow lines of $\mathrm{H}_{2} \mathrm{O}$ and $\mathrm{CO}$ should be the easiest to observe due to the large abundance of these molecules. At the typical densities of protoplanetary disks, water and $\mathrm{CO}$ condensate at temperatures between 130 and $150 \mathrm{~K}$ and 18 and $28 \mathrm{~K}$, respectively [15,25]. At the temperatures of the HD 163296 disk (Fig. 3), the water snow line occurs within 3 A.U. from the star and is not resolved by our observations. Instead, the $\mathrm{CO}$ snow line might occur between 40 and 180 A.U. and might overlap with the ringed structures observed in the continuum.

A key aspect of the sintering and frost line models is that the formation of continuum emission rings by evaporation of volatile elements does not require and might not produce 


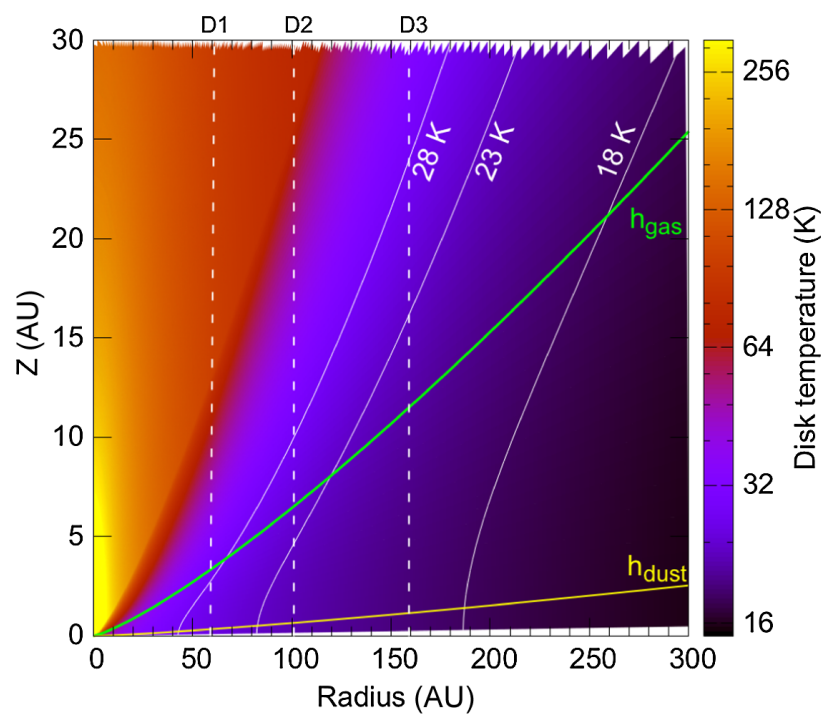

FIG. 3. Temperature of the HD 163296 disk inferred from the observations of the $\mathrm{CO}$ emission. The white solid lines correspond to temperatures of 18,23 , and $28 \mathrm{~K}$, and indicate the range of condensation temperatures for $\mathrm{CO}$ molecules. The vertical dashed line indicates the location of the dust gaps. The green and yellow curves indicate the pressure scale height of gas $\left(h_{\text {gas }}\right)$ and of large dust grains $\left(h_{\text {dust }}\right)$, respectively.

any variation in the gas distribution. Actually, these models require a constant radial inflow of particles that does not happen if the gas density has bumps in the radial direction. Therefore, the depletion of $\mathrm{CO}$ molecules measured inside the middle and outer gaps is inconsistent with the evaporation of volatile elements. However, this process might play a role in shaping the disk regions around the innermost dust gap at 59 A.U., where the $\mathrm{CO}$ observations do not show any evidence of gas depletion. Future ALMA observations of molecules capable of tracing denser gas, e.g., $\mathrm{HCO}^{+}$[45], are required to set more stringent constraints on the nature of this inner gap and on the role of the evaporation of volatile elements.

Conclusions.-We have presented ALMA observations of the HD 163296 circumstellar disk that reveal three dark rings in the dust continuum emission. HD 163296 is the third system known to host multiple rings in the dust continuum emission, but it is the only one for which we have succeeded in mapping the molecular gas emission at the same angular resolution of the continuum. By comparing the observations with radiative transfer models, we find that the continuum rings can be explained with the presence of three circular dust depleted gaps with radii of 60, 100, and 160 A.U. Furthermore, we find that the density of CO molecules inside the middle and outer dust gaps is also reduced compared to the surrounding regions.

We argue that the morphology of these two gaps is consistent with gravitational perturbations caused by two Saturn-mass planets orbiting at about 100 and 160 A.U. Such large orbital radii and the young age of this disk imply that giant planets might form much farther away from the central star and much more rapidly than previously thought. Finally, we note that the presented planet configuration might not be unique. A detailed analysis of the HD 163296 ALMA observations in the framework of planet-disk interaction models will be presented in Liu et al. [46].

We do not find any evidence of gas depletion inside the inner dust gap, although, due to the large optical depth of the observed CO lines, we cannot rule out the presence of a shallow gas gap. The morphology of the inner dust gap is not well described by perturbations caused by a single planet. Instead we suggest that it might have formed as the result of opacity variations at the outer edge of a MRI dead zone or at the $\mathrm{CO}$ frost line.

The results presented in this Letter highlight the importance of imaging protoplanetary disks in both the dust and molecular line emission at high angular resolution. Thanks to the large flux and radial extent, the HD 163296 disk is a perfect target for future ALMA follow-up observations. Furthermore, differently from HL Tau, the relative isolation of the HD 163296 disk enables optical and infrared observations to map the morphology of the disk surface and, perhaps, directly detect forming planets.

We thank Munetake Momose, Misato Fukagawa, and Giovanni Rosotti for the helpful conversation. This paper makes use of the following Atacama Large Millimeter and Submillimeter Array (ALMA) data: ADS/ JAO.ALMA\#2013.1.00601.S. ALMA is a partnership of European Southern Observatory (ESO) (representing its member states), National Science Foundation (USA), and National Institutes of Natural Sciences (Japan), together with National Research Council (Canada), National Science Council and Academia Sinica Institute of Astronomy and Astrophysics (Taiwan), and Korea Astronomy and Space Science Institute (Korea), in cooperation with Chile. The Joint ALMA Observatory is operated by ESO, Associated Universities, Inc/National Radio Astronomy Observatory (NRAO), and National Astronomical Observatory of Japan. The National Radio Astronomy Observatory is a facility of the National Science Foundation operated under cooperative agreement by Associated Universities, Inc. A. I. and Y. B. acknowledge support from the NSF Grant No. AST-1535809 and the National Aeronautics and Space Administration Grant No. NNX15AB06G. E. W. acknowledge support from the NRAO Student Observing Support Grant No. AST0836064. H. L. and S. L. acknowledge the support from the Laboratory Directed Research and Development program at Los Alamos National Laboratory. C. F. M. gratefully acknowledges an European Space Agency Research Fellowship. Part of this research was carried out at the Jet Propulsion Laboratory, California Institute of Technology, under a contract with the National Aeronautics and Space Administration. 
*isella@ rice.edu

[1] M. Mayor and D. Queloz, Nature (London) 378, 355 (1995).

[2] A. W. Howard, G. W. Marcy, J. A. Johnson, D. A. Fischer, J. T. Wright, H. Isaacson, J. A. Valenti, J. Anderson, D. N. C. Lin, and S. Ida, Science 330, 653 (2010).

[3] M. Mayor, M. Marmier, C. Lovis, S. Udry, D. Ségransan, F. Pepe, W. Benz, J. Bertaux, F. Bouchy, X. Dumusque, G. Lo Curto, C. Mordasini, D. Queloz, and N. C. Santos, arXiv: 1109.2497 [Astron. Astrophys. (to be published)].

[4] W. J. Borucki et al., Astrophys. J. 736, 19 (2011).

[5] T. D. Morton, S. T. Bryson, J. L. Coughlin, J. F. Rowe, G. Ravichandran, E. A. Petigura, M. R. Haas, and N. M. Batalha, Astrophys. J. 822, 86 (2016).

[6] R. Helled, P. Bodenheimer, M. Podolak, A. Boley, F. Meru, S. Nayakshin, J. J. Fortney, L. Mayer, Y. Alibert, and A. P. Boss, Protostars and Planets VI, 643 (2014).

[7] C. L. Brogan et al. (ALMA Partnership), Astrophys. J. Lett. 808, L3 (2015).

[8] S. M. Andrews, D. J. Wilner, Z. Zhu, T. Birnstiel, J. M. Carpenter, L. M. Pérez, X.-N. Bai, K. I. Öberg, A. M. Hughes, A. Isella, and L. Ricci, Astrophys. J. Lett. 820, L40 (2016).

[9] P. Barge and J. Sommeria, Astron. Astrophys. 295, L1 (1995).

[10] H. H. Klahr and T. Henning, Icarus 128, 213 (1997).

[11] H. Li, S. A. Colgate, B. Wendroff, and R. Liska, Astrophys. J. 551, 874 (2001).

[12] W. Lyra, A. Johansen, H. Klahr, and N. Piskunov, Astron. Astrophys. 491, L41 (2008).

[13] W. Lyra and M.-K. Lin, Astrophys. J. 775, 17 (2013).

[14] W. Fu, H. Li, S. Lubow, S. Li, and E. Liang, Astrophys. J. Lett. 795, L39 (2014).

[15] K. Zhang, G. A. Blake, and E. A. Bergin, Astrophys. J. Lett. 806, L7 (2015).

[16] S. Okuzumi, M. Momose, S.-i. Sirono, H. Kobayashi, and H. Tanaka, Astrophys. J. 821, 82 (2016).

[17] G. Bryden, X. Chen, D. N. C. Lin, R. P. Nelson, and J. C. B. Papaloizou, Astrophys. J. 514, 344 (1999).

[18] P. Pinilla, M. Benisty, and T. Birnstiel, Astron. Astrophys. 545, A81 (2012).

[19] Z. Zhu, R. P. Nelson, R. Dong, C. Espaillat, and L. Hartmann, Astrophys. J. 755, 6 (2012).

[20] S. Jin, S. Li, A. Isella, H. Li, and J. Ji, Astrophys. J. 818, 76 (2016).

[21] M. E. van den Ancker, P. S. The, H. R. E. Tjin A Djie, C. Catala, D. de Winter, P. F. C. Blondel, and L. B. F. M. Waters, Astron. Astrophys. 324, L33 (1997).

[22] A. Isella, L. Testi, A. Natta, R. Neri, D. Wilner, and C. Qi, Astron. Astrophys. 469, 213 (2007).
[23] I. de Gregorio-Monsalvo et al., Astron. Astrophys. 557, A133 (2013).

[24] G. Guidi et al., Astron. Astrophys. 588, A112 (2016).

[25] C. Qi, K. I. Öberg, S. M. Andrews, D. J. Wilner, E. A. Bergin, A. M. Hughes, M. Hogherheijde, and P. D'Alessio, Astrophys. J. 813, 128 (2015).

[26] See Supplemental Material at http://link.aps.org/ supplemental/10.1103/PhysRevLett.117.251101 for quantitative information related to the observed dust and gas emission, and an extensive description of the analysis and modeling procedures.

[27] K. A. Rosenfeld, S. M. Andrews, A. M. Hughes, D. J. Wilner, and C. Qi, Astrophys. J. 774, 16 (2013).

[28] T. L. Wilson, Rep. Prog. Phys. 62, 143 (1999).

[29] J. C. B. Papaloizou, R. P. Nelson, W. Kley, F. S. Masset, and P. Artymowicz, Protostars and Planets V, 655 (2007).

[30] G. P. Rosotti, A. Juhasz, R. A. Booth, and C. J. Clarke, Mon. Not. R. Astron. Soc. 459, 2790 (2016).

[31] S. Wolf, A. Moro-Martín, and G. D’Angelo, Planet. Space Sci. 55, 569 (2007).

[32] K. D. Kanagawa, T. Muto, H. Tanaka, T. Tanigawa, T. Takeuchi, T. Tsukagoshi, and M. Momose, Publ. Astron. Soc. Jpn. 68, 43 (2016).

[33] K. D. Kanagawa, T. Muto, H. Tanaka, T. Tanigawa, T. Takeuchi, T. Tsukagoshi, and M. Momose, Astrophys. J. Lett. 806, L15 (2015).

[34] N. I. Shakura and R. A. Sunyaev, Astron. Astrophys. 24, 337 (1973).

[35] D. Tamayo, A. H. M. J. Triaud, K. Menou, and H. Rein, Astrophys. J. 805, 100 (2015).

[36] S. A. Balbus and J. F. Hawley, Astrophys. J. 376, 214 (1991).

[37] E. Chiang and R. Murray-Clay, Nat. Phys. 3, 604 (2007).

[38] J. Igea and A. E. Glassgold, Astrophys. J. 518, 848 (1999).

[39] D. Perez-Becker and E. Chiang, Astrophys. J. 735, 8 (2011).

[40] T. Umebayashi and T. Nakano, Publ. Astron. Soc. Jpn. 33, 617 (1981).

[41] K. A. Miller and J. M. Stone, Astrophys. J. 534, 398 (2000).

[42] J. B. Simon, P. J. Armitage, and K. Beckwith, Astrophys. J. 743, 17 (2011).

[43] M. Flock, J. P. Ruge, N. Dzyurkevich, T. Henning, H. Klahr, and S. Wolf, Astron. Astrophys. 574, A68 (2015).

[44] A. Banzatti, P. Pinilla, L. Ricci, K. M. Pontoppidan, T. Birnstiel, and F. Ciesla, Astrophys. J. Lett. 815, L15 (2015).

[45] H.-W. Yen, H. B. Liu, P.-G. Gu, N. Hirano, C.-F. Lee, E. Puspitaningrum, and S. Takakuwa, Astrophys. J. Lett. 820, L25 (2016).

[46] S.-F. Liu, S. Jin, S. Li, H. Li, and A. Isella (to be published). 